\title{
The spatial and temporal fidelity in real-time MRI in patients with sinus rhythm and arrhythmias
}

\author{
Francisco Contijoch*, Kelly Rogers, Walter R Witschey, Robert C Gorman, Yuchi Han \\ From 17th Annual SCMR Scientific Sessions \\ New Orleans, LA, USA. 16-19 January 2014
}

\begin{abstract}
Background
Irregularly irregular arrhythmia is difficult to image with conventional cine CMR because arrhythmia rejection and registration-based reconstruction result in cardiac motion artifacts or unachievable breath-hold durations. Undersampled radial trajectories, combined with a suitable non-Cartesian reconstruction algorithm, have the potential to overcome these problems by sampling a small number of radial projections without loss of temporal fidelity associated with temporal regularization. Despite the large number of available real-time reconstruction algorithms and sampling trajectories, there are very few reports regarding optimal values for basic reconstruction parameters such as image exposure time (radial projections per image frame) and temporal resolution (view sharing). Our goal was to address the problem of spatial and temporal fidelity in real-time MRI using automatic active contour segmentation in subjects with sinus rhythm and also patients with irregularly irregular arrhythmias.
\end{abstract}

\section{Methods}

Short-axis stacks of images were acquired in 20 subjects using standard retrospective gated bSSFP cine imaging and a golden-angle radial bSSFP trajectory (total views = 4000 , views per image $=34$ ) reconstructed into $128 \times$ 128 images via conjugate gradient-SENSE based iterative reconstruction (Gadgetron). Golden-angle radial acquisitions can be reconstructed with a variable number of projections (quantified as the exposure time Te of each frame). In addition, projections can be shared between two adjacent frames resulting in higher reconstructed temporal resolution. Two datasets were reconstructed with varying exposure time $(\mathrm{Te}=28-840 \mathrm{~ms})$ and temporal resolution $(\mathrm{Tr}=2.8-96.2 \mathrm{~ms})$ to evaluate the effect on ejection fraction (EF). Global function was assessed via semi-automated segmentation of all phases and all left ventricular slices (ITK-SNAP, Philadelphia PA) in the short-axis stack and EDV, ESV, SV, and EF were measured.

\section{Results}

EF is sensitive to both exposure time and temporal resolution and we found an $\mathrm{Te}<100 \mathrm{~ms}$ and $\mathrm{Tr}<22.8 \mathrm{~ms}$ is necessary to avoid substantial error. The figure illustrates how errors accumulate beyond these values. With these values $(\mathrm{Te}=95.2 \mathrm{~ms}, \mathrm{Tr}=2.8 \mathrm{~ms})$, we did not observe significant differences in EF (real time $=46.7 \pm$ $20.1 \%$, cine $=47.2 \pm 21.9 \% \mathrm{p}=0.6282$,) or stroke volume (real time $=62.0 \pm 17.8 \%$, cine $=64.3 \pm 23.9 \%$, $\mathrm{p}=0.4746)$ in 11 subjects with sinus rhythm. In 9 subjects with rhythm disturbances, volume curves (illustrated in the Figure 1) were obtained which show the effect of arrhythmic beats. EF quantified in these patients, the estimated EF was higher than that from low quality CINE images.

\section{Conclusions}

Accurate volume assessment at high spatiotemporal fidelity is possible in patients with irregular arrhythmias with real-time CMR and active contour segmentation. Our estimated values for optimal image exposure time 

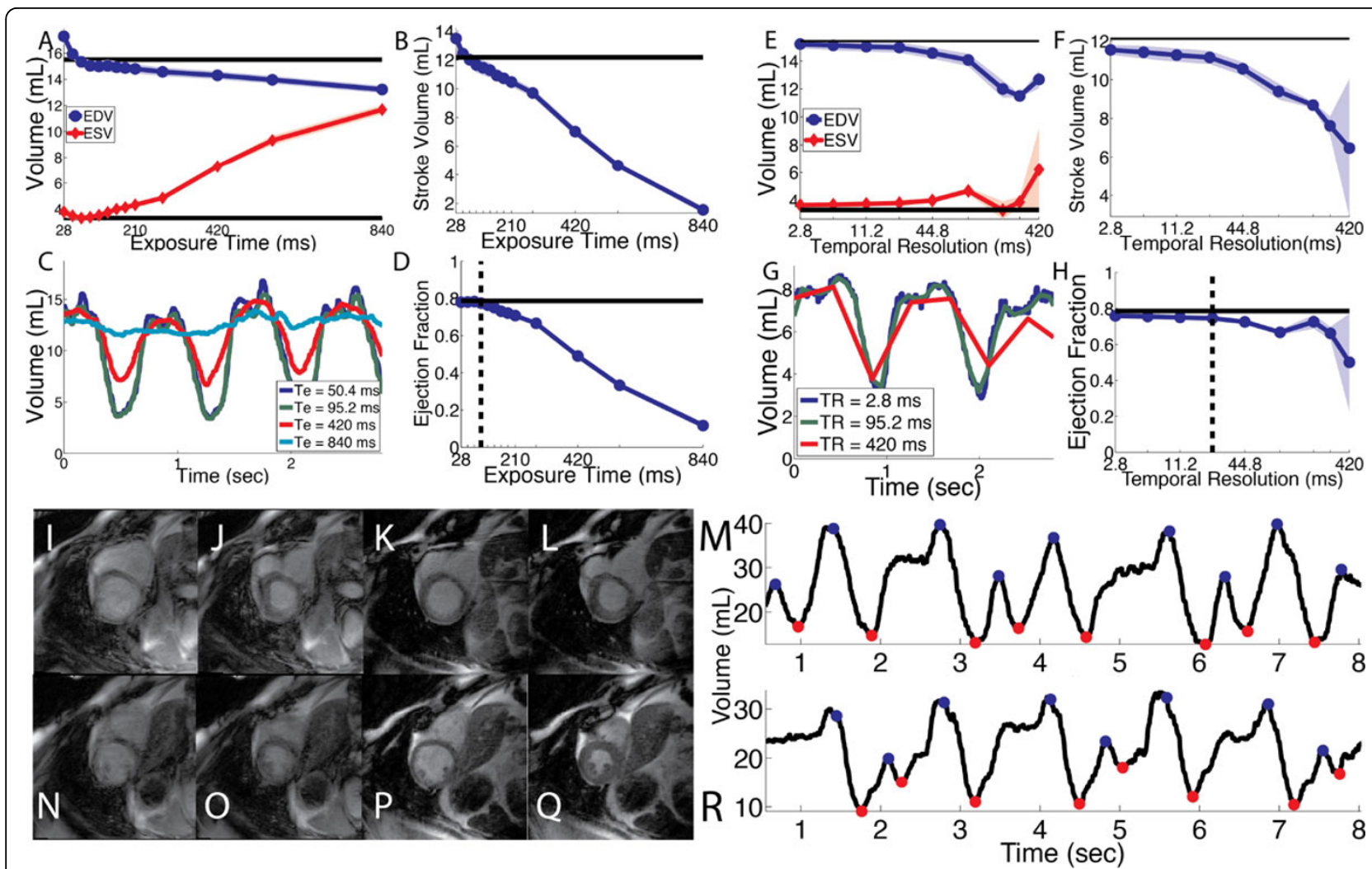

Figure 1 Panel A-D illustrate the effect of exposure time on measure volumetric values. Increasing the exposure time improves k-space sampling but results in temporal blurring. Panels E-H illustrate the effect of reconstructed frame rate on measure values. As the frame rate decreases, the local maxima and minima values are not obtained leading to changes in hemodynamic evaluation. The proposed method was utilized to image patients with frequent irregular arrhythmias. The improvement in image quality is shown for two different slice locations Panel I-L and N-Q. Standard EKG-gated images are shown at end-diastole (I and N) and end-systole (J and O). The real-time method shows improved image quality at both phases (end-diastole: $K$ and $P$, end systole: $L$ and $Q$ ). Furthermore, by obtaining volume for 8 seconds, the occurrence of premature beats can be observed ( $\mathrm{M}$ and $\mathrm{R}$ ). End-diastolic points are labeled blue and end-systolic points are labeled with a red dot.

and temporal resolution will guide future consensus regarding these basic reconstruction parameters.

\section{Funding}

K99-HL108157, R01-HL103723, T32HL007954, T32EB009384.

Published: 16 January 2014

doi:10.1186/1532-429X-16-S1-011

Cite this article as: Contijoch et al:: The spatial and temporal fidelity in real-time MRI in patients with sinus rhythm and arrhythmias. Journal of Cardiovascular Magnetic Resonance 2014 16(Suppl 1):011.
Submit your next manuscript to BioMed Central and take full advantage of:

- Convenient online submission

- Thorough peer review

- No space constraints or color figure charges

- Immediate publication on acceptance

- Inclusion in PubMed, CAS, Scopus and Google Scholar

- Research which is freely available for redistribution 